\title{
Contribution of Neuraminidase of Influenza Viruses to the Sensitivity to Sera Inhibitors and Reassortment Efficiency
}

\author{
Irina Kiseleva ${ }^{\wedge} *$, Natalie Larionova ${ }^{\wedge}$, Ekaterina Fedorova, Ekaterina Bazhenova, Irina Dubrovina, \\ Irina Isakova-Sivak and Larisa Rudenko
}

Department of Virology, Institute of Experimental Medicine, St. Petersburg, Russia

\begin{abstract}
Live attenuated influenza vaccine (LAIV) represent reassortant viruses with hemagglutinin (HA) and neuraminidase (NA) gene segments inherited from circulating wild-type (WT) parental influenza viruses recommended for inclusion into seasonal vaccine formulation, and the 6 internal protein-encoding gene segments from cold-adapted attenuated master donor viruses (genome composition 6:2). In this study, we describe the obstacles in developing LAIV strains while taking into account the phenotypic peculiarities of WT viruses used for reassortment. Genomic composition analysis of 849 seasonal LAIV reassortants revealed that over $80 \%$ of reassortants based on inhibitor-resistant WT viruses inherited WT NA, compared to $26 \%$ of LAIV reassortants based on inhibitor-sensitive WT viruses. In addition, the highest percentage of LAIV genotype reassortants was achieved when WT parental viruses were resistant to non-specific serum inhibitors. We demonstrate that NA may play a role in influenza virus sensitivity to non-specific serum inhibitors. Replacing NA of inhibitor-sensitive WT virus with the NA of inhibitor-resistant master donor virus significantly decreased the sensitivity of the resulting reassortant virus to serum heat-stable inhibitors.
\end{abstract}

Keywords: Influenza virus, live influenza vaccine, neuraminidase, normal serum inhibitors, reassortment.

\section{INTRODUCTION}

The Russian live attenuated influenza vaccine (LAIV) consists of reassortant viruses, which contain hemagglutinin (HA) and neuraminidase (NA) gene segments from circulating wild-type (WT) viruses which are recommended on annual basis for inclusion in a seasonal vaccine formulation on a background of internal genes derived from the attenuated vaccine master donor viruses (MDVs), A/Leningrad/134/17/57 (H2N2) or B/USSR/60/69 (representing genomic composition of $6 \mathrm{MDV}$ genes and 2 WT genes). LAIV strains possess the cold-adapted, temperature-sensitive and attenuated phenotypes from the MDVs. According to the Russian regulatory practice, 5:3 genomic composition is also acceptable for LAIV [1] if the third WT gene of the vaccine candidate does not encode RNA polymerase protein complex components.

Based on ongoing world-wide surveillance of influenza virus circulation, the World Health Organization (WHO) annually recommends viruses that antigenically match to circulating strains in order to be included in influenza vaccines [2]. As the time for vaccine virus generation is extremely limited, and because only $6: 2$ and 5:3 reassortant

\footnotetext{
*Address correspondence to this author at the Institute of Experimental Medicine, 12 Acad. Pavlov Street, St. Petersburg, Russia 197376;

Tel: +7 (812) 234 6860; Fax: +7(812)2349489;

E-mail: irina.v.kiseleva@gmail.com

${ }^{\wedge}$ These authors contributed equally to this work.
}

virus ratios are allowed as vaccine candidates, the methods for vaccine development must be rapid, reproducible, and reliable. The most successful and well-established current strategy for preparing attenuated reassortant vaccine virus has been to co-infect embryonated chicken eggs with equal doses of selected WT virus with cold-adapted (CA) MDV. Co-infection with MDV and WT viruses theoretically may yield as many as 256 combinations of gene segments upon gene reassortment, and, thus, 256 genetically different genotypes. To diminish the number of undesired reassortant viruses arising during co-infection, two selecting factors serum raised against the MDV and low incubation temperature $\left(25-26{ }^{\circ} \mathrm{C}\right)$ - are used in subsequent selective passages $[3,4]$.

Successful and quick generation of LAIV reassortants depends considerably on a variety of factors, including the phenotypic properties of the WT parental strain. Recently, many strains identified by the WHO as those likely to resemble the strains in circulation during the next epidemic season have been found to be highly sensitive to non-specific serum inhibitors $[5,6]$. Sera and other biological liquids of different animal species contain non-specific inhibitors of influenza viruses. Composition and concentration of inhibitors depends on animal species [7, 8]. Mechanism of interaction of different inhibitor classes with influenza virus is described in set of studies [7-9]. The standard protocol for developing LAIV reassortants includes treating anti-MDV serum with receptor destroying enzyme (RDE) to remove 
non-specific inhibitors. However, properly treated serum may still contain residual levels of heat-stable inhibitors. The presence of residual non-specific inhibitors in immune antiMDV serum may alter the antibody recognition pattern and complicate development of reassortant vaccine strains based on inhibitor-sensitive WT viruses. Additionally, some early publications debated whether the LAIV candidate should be inhibitor-sensitive or resistant. Some researchers believed that serum inhibitor-resistant LAIV candidates are more attenuated and, therefore, more attractive for human use [10].

In this paper, we analyzed 849 reassortants of several WT viruses with MDVs obtained using classical egg-based reassortment techniques. We describe some aspects of developing LAIV reassortants, including obstacles in development, importance of WT parental virus properties, and differences in how seasonal human influenza viruses and H5N1 avian influenza viruses reassort with MDVs.

\section{MATERIALS AND METHODOLOGY}

\section{Viruses}

A total of 44 influenza A and B viruses were chosen for this study. Of these, 37 WT influenza A and B viruses were obtained from the Centers for Disease Control and Prevention (CDC, Atlanta, GA, USA). Reassortant vaccine candidates for parenterally administered, inactivated influenza vaccine of subtypes H5N1 (NIBRG-23, INDO/05, VN1203), H3N2 (NIB-64), and H1N1 (IRV-148) based on $\mathrm{A} / \mathrm{PR} / 8 / 34$ (H1N1) strain (PR8) as the high-yield internal gene donor were generated by reverse genetics techniques from A/turkey/Turkey/1/05 (H5N1), A/Indonesia/5/05 (H5N1), A/Vietnam/1203/2004 (H5N1), A/Perth/16/09 (H3N2), and A/Brisbane/59/07 (H1N1) influenza viruses, respectively. The cleavage site of genes coding HA of H5N1 WT viruses was modified by removing 4 basic amino acid codons. PR8-based reassortants were obtained from CDC and WHO. Two cold-adapted MDVs, A/Leningrad/134/ $17 / 57$ (H2N2) (A-MDV) and B/USSR/60/69 (B-MDV), that are used to develop the Russian LAIV are the property of the Institute of Experimental Medicine (St. Petersburg, Russia).

Viruses were propagated in 10-11-day-old embryonated chicken eggs at $32-33^{\circ} \mathrm{C}$.

\section{Genetic Reassortment Procedures}

MDV and WT viruses were co-infected into embryonated chicken eggs by classical reassortment techniques as described previously [3]. Briefly, 5-6 rounds of selective propagation were performed, 3 of them at $25-26{ }^{\circ} \mathrm{C}$. The production and selection of reassortants were carried out in the presence of rabbit or rat RDE-treated MDV antiserum. Cloning by endpoint dilution was performed in each of the last 3-4 passages.

\section{RNA Isolation}

RNA was isolated from influenza virus-infected allantoic fluid using a QIAamp Viral RNA minikit per manufacturer's instructions (Qiagen GmbH, Hilden, Germany).

\section{Genomic Composition Analysis}

The parental origin of the RNA segments of each reassortant virus was monitored by restriction fragment length polymorphism (RFLP) analysis [11], or determined by mixed PCR assays [12]. Nucleotide sequencing was performed using a BigDye Terminator v3.1 cycle sequencing kit and a 3130xl Genetic Analyzer (both from Applied Biosystems, Carlsbad, CA, USA) according to manufacturer's instructions. Multiple sequence alignment analysis was performed using Lasergene version 7.1 sequence analysis software.

\section{Hemagglutination Inhibition (HAI) Assays}

HAI assays were performed using standard techniques as described in [13], in 96-well microtiter plates at room temperature with $1.0 \%$ suspension of chicken or human $0(\mathrm{I})$ $\mathrm{Rh}^{+}$red blood cells.

\section{Sensitivity to Non-Specific Serum Inhibitors}

To analyze the sensitivity of influenza viruses to nonspecific inhibitors, normal guinea pig serum (Rappolovo Laboratory Animals Farm, St. Petersburg, Russia) was used. Serum was heat-inactivated for $10 \mathrm{~min}$ at $80{ }^{\circ} \mathrm{C}$ to eliminate temperature-sensitive inhibitors, and used for HAI assays with 4 hemagglutinating units of each viruses. A virus was considered to be resistant to non-specific serum inhibitors if the HAI titer was $\leq 40$, and designated as sensitive to nonspecific serum inhibitors if HAI titer was $\geq 80$.

\section{Statistical Analyses}

Pearson chi-square $\left(\chi^{2}\right)$ test was used to identify associations between inhibitor sensitivity of parental viruses used for reassortment and reassortment efficiency. Student's $t$ distribution test for dependent samples was used to evaluate the differences in geometrical mean HAI titers between groups of viruses compared for their ability to react with normal guinea pig sera. A value of $\mathrm{p}<0.001$ was considered significant, and $\mathrm{p}<0.0001$ was considered highly significant.

\section{RESULTS}

\section{Reassortment of Seasonal WT Influenza Viruses with MDVs for LAIV Generation}

Most influenza A and B viruses circulating prior to the year 2000 readily reassorted with MDVs, and reassortants with the desired vaccine genomic composition of $6: 2$ and 5:3 were easy to generate. The resulting reassortants typically inherited $60-90 \%$ of internal genes from MDVs, and 90$100 \%$ HA and NA genes from WT parents. In contrast, vaccine strains based on WT viruses circulating after year 2000 were much more difficult to develop. In our experience only $11-14 \%$ of reassortants derived from post-2000 viruses using standard techniques possessed NA of WT parental virus. The percentage of internal genes derived from MDVs, especially $M$ and NS, was also relatively low. WT- and MDV-gene segment distribution in the genome of reassortants generated on the basis of some influenza $\mathrm{A}$ and B viruses circulating in various years is presented in Table $\mathbf{1}$. 
Table 1. Gene segment distribution in the genomes of reassortants generated from various influenza A and B viruses circulating in different years.

\begin{tabular}{|c|c|c|c|c|c|c|c|c|}
\hline \multicolumn{9}{|c|}{ Influenza A Viruses } \\
\hline \multirow[b]{2}{*}{ Gene Segments } & \multicolumn{4}{|c|}{ Before $2000^{1}$} & \multicolumn{4}{|c|}{ After $2000^{2}$} \\
\hline & $\%$ & (n) & $\%$ & (n) & $\%$ & (n) & $\%$ & (n) \\
\hline PB2 & $2.1 \%$ & (2) & $97.9 \%$ & (95) & $9.6 \%$ & (5) & $90,4 \%$ & $(47)$ \\
\hline PB1 & $5,2 \%$ & (5) & $94,8 \%$ & $(92)$ & $40,4 \%$ & (21) & $59,6 \%$ & (31) \\
\hline HA & $100,0 \%$ & (97) & $0,0 \%$ & $(0)$ & $100,0 \%$ & $(52)$ & $0,0 \%$ & $(0)$ \\
\hline NP & $29,9 \%$ & (29) & $70,1 \%$ & $(68)$ & $44,2 \%$ & (23) & $55,8 \%$ & $(29)$ \\
\hline NA & $91,8 \%$ & (89) & $8,2 \%$ & (8) & $13,5 \%$ & (7) & $86,5 \%$ & (45) \\
\hline M & $42,3 \%$ & (41) & $57,7 \%$ & (56) & $65,4 \%$ & (34) & $34,6 \%$ & (18) \\
\hline NS & $47,4 \%$ & $(46)$ & $52,6 \%$ & (51) & $80,8 \%$ & $(42)$ & $19,2 \%$ & $(10)$ \\
\hline Gene Segments & \multicolumn{2}{|c|}{ WT } & \multicolumn{2}{|c|}{ MDV } & \multicolumn{2}{|c|}{ WT } & \multicolumn{2}{|c|}{ MDV } \\
\hline PB2 & $8.3 \%$ & (1) & $91.7 \%$ & (11) & $19.9 \%$ & (48) & $80.1 \%$ & (193) \\
\hline PB1 & $17,0 \%$ & (2) & $83,0 \%$ & (10) & $39,8 \%$ & (96) & $60,2 \%$ & (145) \\
\hline PA & $16,7 \%$ & $(2)$ & $83,3 \%$ & (10) & $10,0 \%$ & (24) & $90,0 \%$ & (217) \\
\hline HA & $100,0 \%$ & (12) & $0,0 \%$ & $(0)$ & $100,0 \%$ & (241) & $0,0 \%$ & $(0)$ \\
\hline NP & $41,7 \%$ & (5) & $58,3 \%$ & (7) & $39,8 \%$ & (96) & $60,2 \%$ & (145) \\
\hline NA & $91,7 \%$ & (11) & $8,3 \%$ & (1) & $11,2 \%$ & (27) & $88,8 \%$ & (214) \\
\hline M & $50,0 \%$ & (6) & $50,0 \%$ & (6) & $90,0 \%$ & (217) & $10,0 \%$ & (24) \\
\hline
\end{tabular}

Influenza A viruses:

${ }^{1}$ Reassortants of A/Leningrad/134/17/57 (H2N2) master donor virus (MDV) with A/Nanchang/933/95 (H3N2), A/Beijing/262/95 (H1N1), A/Perth/13/95 (H1N1), A/Johannesburg/82/96 (H1N1), A/Panama/2007/99 (H3N2), A/New Caledonia/20/99 (H1N1)

${ }^{2}$ Reassortants of A/Leningrad/134/17/57 (H2N2) MDV with A/Wellington/01/04 (H3N2), A/Malaysia/01/04 (H3N2), and A/Brisbane/10/07 (H3N2). Influenza B viruses:

${ }^{3}$ Reassortants of B/USSR/60/69 MDV with B/Harbin/07/94, B/Shandong/7/97

${ }^{4}$ Reassortants of B/USSR/60/69 MDV with B/Florida/4/06, B/Brisbane/3/07, B/Bangladesh/1994/10, and B/Wisconsin/1/10

The difficulties encountered in generating current LAIV reassortant viruses led us to perform a large-scale analysis of reassortment efficiency. We found that the rate of NA inheritance by reassortant viruses depended on the inhibitor sensitivity of the WT parental virus to normal guinea pig serum regardless of its specific type/subtype, with the exception of subtype H5N1 viruses. Table 2 shows the distribution of reassortants bearing NA gene segments from WT or cold-adapted parental viruses stratified by the inhibitor sensitivity of WT parental viruses. In fact, over $80 \%$ of all reassortants based on inhibitor-resistant WT viruses (297 out of 356) inherited WT NA, compared to only $26 \%$ of reassortants based on inhibitor-sensitive WT viruses (130 out of 493; Fig. 1). The rate of WT NA inheritance by influenza virus reassortants of various types/subtypes varied, but generally was significantly higher when parental viruses were inhibitor-resistant (Table 3).

Genomic analysis of 849 reassortants revealed that the highest percentage of $6: 2$ and 5:3 vaccine-appropriate genotype reassortants was achieved when using WT parental viruses resistant to non-specific serum inhibitors (Fig. 2). When using inhibitor-resistant group of influenza viruses, vaccine-appropriate reassortants (with either 6:2 and 5:3 gene composition) were found in the 108/136 (79\%; H1N1 subtype), $5 / 5$ (100\%; H2N2 subtype), 32/74 (43\%; H3N2 subtype), and 51/88 (58\%; type B) cases. In contrast, when using inhibitor-sensitive WT parental viruses, only 4/29 (14\%; H2N2 subtype), 25/126 (20\%; H3N2 subtype), and $24 / 326$ (7\%; type B) vaccine reassortants were isolated (Table 3). Since only 6:2 and 5:3 genomic combinations are appropriate for generating LAIV strains, we correlated the number of reassortants with vaccine genomic composition and all other genomic combinations. Collected data were analyzed by Pearson $\chi^{2}$ test (Table 4). Reassortants with 6:2 or 5:3 genomic composition were generated significantly more frequently from inhibitor-resistant WT influenza viruses $\left(\chi^{2}=195.78 ; \mathrm{p}<0.0001\right)$. 
Table 2. Rate of neuraminidase (NA) gene segment inheritance by reassortants of master donor viruses (MDV) with wild-type (WT) influenza viruses of varying sensitivity to non-specific, heat-stable inhibitors in guinea pig serum.

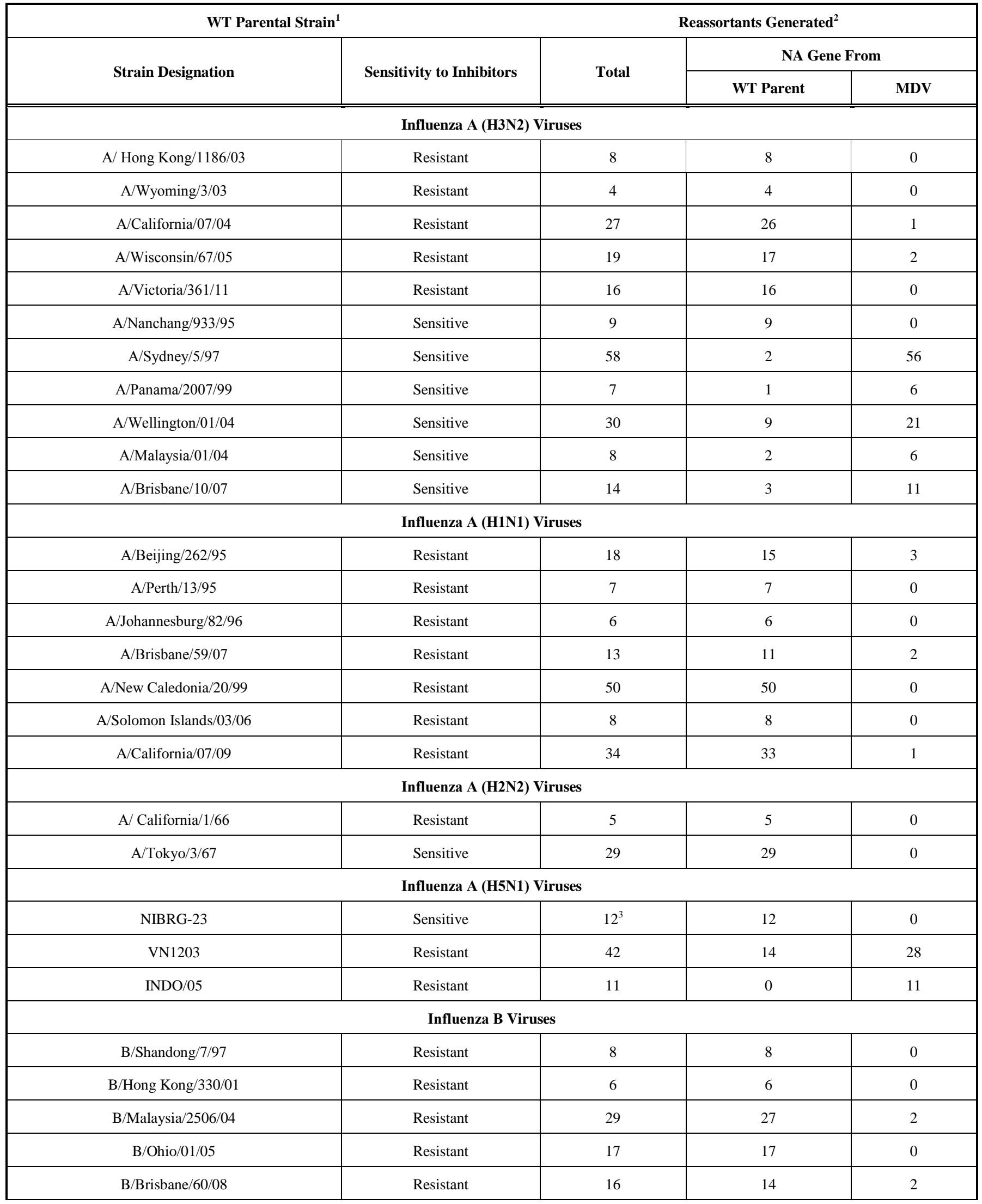


Table 2. Contd....

\begin{tabular}{|c|c|c|c|c|}
\hline \multicolumn{2}{|c|}{ WT Parental Strain ${ }^{1}$} & \multicolumn{3}{|c|}{ Reassortants Generated $^{2}$} \\
\hline \multirow{2}{*}{ Strain Designation } & \multirow{2}{*}{ Sensitivity to Inhibitors } & \multirow{2}{*}{ Total } & \multicolumn{2}{|c|}{ NA Gene From } \\
\hline & & & WT Parent & MDV \\
\hline \multicolumn{5}{|c|}{ Influenza B Viruses } \\
\hline B/Texas/26/08 & Resistant & 12 & 5 & 7 \\
\hline B/Petersburg/92/95 & Sensitive & 8 & 3 & 5 \\
\hline B/Johannesburg/05/99 & Sensitive & 14 & 5 & 9 \\
\hline B/Jilin/20/03 & Sensitive & 10 & 4 & 6 \\
\hline $\mathrm{B} /$ Brisbane/3/07 & Sensitive & 13 & 1 & 12 \\
\hline B/Wisconsin/1/10 & Sensitive & 140 & 4 & 136 \\
\hline B/Bangladesh/1994/10 & Sensitive & 57 & 19 & 38 \\
\hline B/Texas/6/11 & Sensitive & 12 & 7 & 5 \\
\hline
\end{tabular}

${ }^{1}$ WT parental virus. ${ }^{2}$ All reassortants inherited HA of WT viruses. ${ }^{3}$ Virus NIBRG-23 (H5N1) did not reassort with MDV when classical reassortment procedures were used; all 12 clones isolated were identical to NIBRG-23 (H5N1) in genomic composition.

\section{Role of NA in Inhibitor Sensitivity of Reassortant Viruses}

We analyzed the following viruses for their reactivity to normal guinea pig serum: LAIV MDVs A/Leningrad/134/ $17 / 57$ (H2N2) and B/USSR/60/69; four influenza A WT viruses; six influenza B WT viruses; six 6:2 reassortants

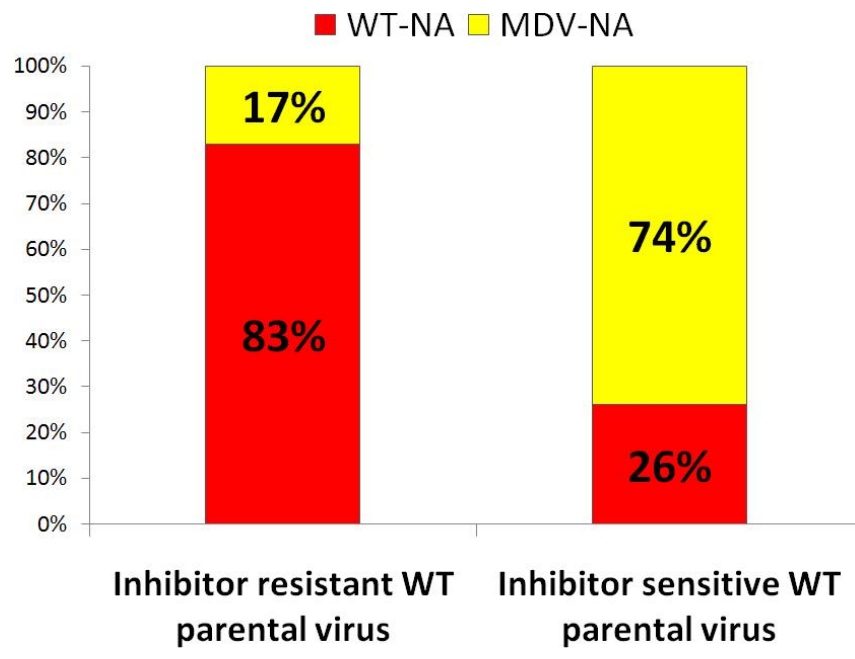

Fig. (1). Rate of NA gene segment inheritance by reassortants of MDVs with WT viruses of varying sensitivity to non-specific serum gamma inhibitors. Left panel: reassortants of A/Leningrad/134/17/57 (H2N2) and B/USSR/60/69 MDVs with inhibitor-resistant WT viruses. Right panel: reassortants of A/Leningrad/134/17/57 (H2N2) and B/USSR/60/69 MDVs with inhibitor-sensitive WT viruses. containing HA and NA from WT virus and internal genes from MDV (R2, R10, R13, R15, R16, R19); and thirteen 7:1 reassortants with a single HA gene segment from the WT

$\square$ Other $\square 5: 3 \quad \square 6: 2$

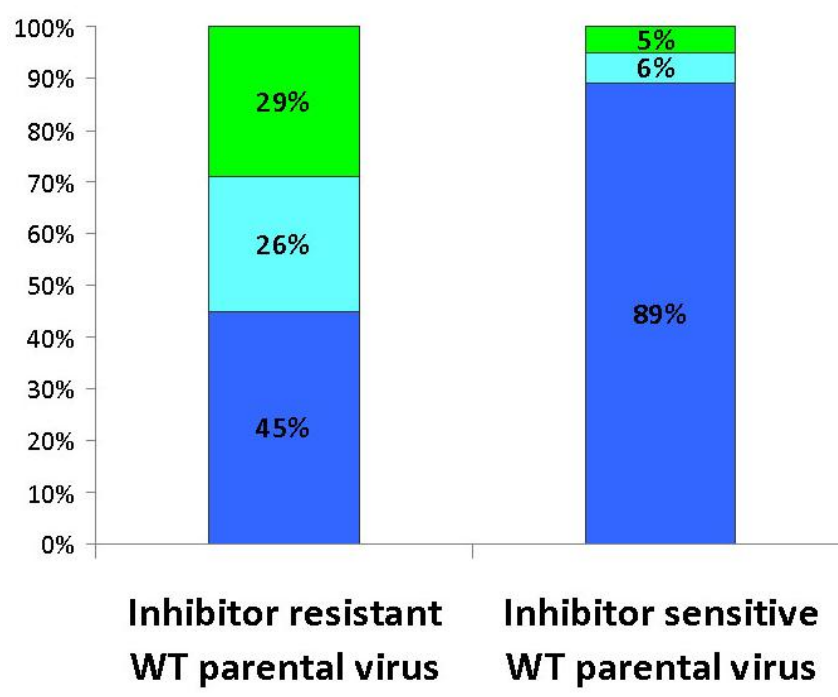

Fig. (2). Genomic composition of reassortment of MDVs with WT viruses of varying sensitivity to non-specific serum gamma inhibitors. Left panel: reassortants of A/Leningrad/134/17/57 (H2N2) and B/USSR/60/69 MDVs with inhibitor-resistant WT viruses. Right panel: reassortants of $\mathrm{A} /$ Leningrad/134/17/57 (H2N2) and B/USSR/60/69 MDVs with inhibitor-sensitive WT viruses. 
Table 3. Genomic composition and rate of NA inheritance by reassortants of MDV and currently circulating influenza viruses of varying sensitivity to non-specific, heat-stable inhibitors in guinea pig serum.

\begin{tabular}{|c|c|c|c|c|c|c|c|c|}
\hline \multicolumn{2}{|c|}{ WT Parental Strain ${ }^{1}$} & \multicolumn{7}{|c|}{ Reassortants Generated $^{2}$} \\
\hline $\begin{array}{c}\text { Number of Viruses } \\
\text { Tested }\end{array}$ & $\begin{array}{l}\text { Sensitivity to } \\
\text { Inhibitors }\end{array}$ & $\begin{array}{c}\text { Total } \\
\text { Number }\end{array}$ & \multicolumn{2}{|c|}{ NA From } & \multicolumn{4}{|c|}{ Genomic Composition ${ }^{3}$} \\
\hline \multicolumn{9}{|c|}{ Influenza A (H3N2) Viruses } \\
\hline 5 & Resistant & 74 & $71(95.9 \%)$ & $3(4.1 \%)$ & $7(9.5 \%)$ & $25(33.8 \%)$ & $1(1.4 \%)$ & $41(55.3 \%)$ \\
\hline \multicolumn{9}{|c|}{ Influenza A (H1N1) $)^{4}$ Viruses } \\
\hline 7 & Resistant & 136 & $130(95.6 \%)$ & $6(4.4 \%)$ & $71(52.2 \%)$ & $37(27.2 \%)$ & 0 & $28(20.6 \%)$ \\
\hline \multicolumn{9}{|c|}{ Influenza A (H2N2) Viruses } \\
\hline 2 & Resistant & 53 & $14(26.4 \%)$ & $39(73.6 \%)$ & 0 & 0 & $39(73.6 \%)$ & $14(26.4 \%)$ \\
\hline 1 & Sensitive & 12 & $12(100 \%)$ & 0 & 0 & 0 & 0 & $12(100 \%)$ \\
\hline \multicolumn{9}{|c|}{ All Influenza A Viruses } \\
\hline 14 & Resistant & 268 & $220(82.1 \%)$ & $48(17.9 \%)$ & $82(30.6 \%)$ & $63(23.5 \%)$ & $40(14.9 \%)$ & $83(31.0 \%)$ \\
\hline 9 & Sensitive & 167 & $67(40.1 \%)$ & $100(59.9 \%)$ & $12(7.2 \%)$ & $17(10.2 \%)$ & $10(6 \%)$ & $128(76.6 \%)$ \\
\hline \multicolumn{9}{|c|}{ All Influenza B Viruses } \\
\hline 6 & Resistant & 88 & $77(87.5 \%)$ & $11(12.5 \%)$ & $21(23.9 \%)$ & $30(34.1 \%)$ & $1(1.1 \%)$ & $36(40.9 \%)$ \\
\hline
\end{tabular}

The complete list of WT parental viruses is presented in Table $1 .{ }^{2}$ All reassortants inherited HA from WT parental viruses. ${ }^{3}$ Genomic composition: $6: 2$ are reassortants containing HA and NA from WT virus; 5:3 are reassortants containing HA and NA, from the WT parent, as well as WT NP, M, or NS gene; 7:1 are single gene reassortants containing only HA from WT virus. ${ }^{4}$ No inhibitor-sensitive WT viruses were found among the type A (H1N1) influenza viruses tested.

influenza virus and 7 RNA segments from MDV (R1, R3$\mathrm{R} 9, \mathrm{R} 11, \mathrm{R} 12, \mathrm{R} 14, \mathrm{R} 17, \mathrm{R} 18)$. In the HAI assay, 6 out of 9 WT parental viruses chosen for this study were highly sensitive to inhibition by normal guinea pig serum (Table 5). The 2 MDVs, A/Leningrad/134/17/57 and B/USSR/60/69, and WT viruses VN1203, INDO/05, and B/Texas/26/08, were highly resistant to inhibitors, as HAI titers did not exceed 10.

The degree of sensitivity (HAI titer) to serum differed dramatically among the 6:2 and 7:1 reassortants generated on the basis on inhibitor-sensitive WT viruses. All 7:1 reassortants possessing HA from inhibitor-sensitive and NA from inhibitor-resistant MDVs were significantly less sensitive to inhibitors than their WT parental viruses or $6: 2$ reassortants $(t=11.09 ; \mathrm{p}<0.00001$; Table 4). While still inhibitor-sensitive, the 7:1 reassortants displayed a $\geq 4$-fold drop in HAI titer with normal guinea pig serum compared to viruses possessing HA and NA from inhibitor-sensitive WT parental virus. For instance, the HAI titer of NIBRG-23 (H5N1) virus, which is highly sensitive to normal guinea pig serum, was 5120. In contrast, the HAI titer of the R3 7:1 reassortant was only 640 when using the same serum.

To determine whether the observed change in inhibitor sensitivity of 7:1 reassortants resulted directly from altered NA function, we compared the sequences of parental viruses and their single gene reassortants. Interestingly, we found identical mutations in the MDV NA gene of several reassortants within type A (nt 765 in R3 and R9) and type B (nt 1085 and nt 1359 in R11 and R17; in R14 and R18) viruses (Table 6). These mutations could be new spontaneous mutations in positions responsible for the adaptation of the virus to chicken eggs, or a minor quasispecies in the initial viral stock. In either case, these mutations did not affect the phenotype of R4 virus, and none of the mutations altered the viruses' sensitivity to heat-stable inhibitors. For instance, R3 was exactly as sensitive as the 2 other NIBRG-23 × A-MDV reassortants, R4 and R5.

Thus, the change in inhibitor sensitivity of $7: 1$ reassortants is likely to result from alterations in NA function rather than mutations. 
Table 4. Statistical analyses of collected data using Pearson chi-square $(\chi 2)$ test and Student's t distribution test.

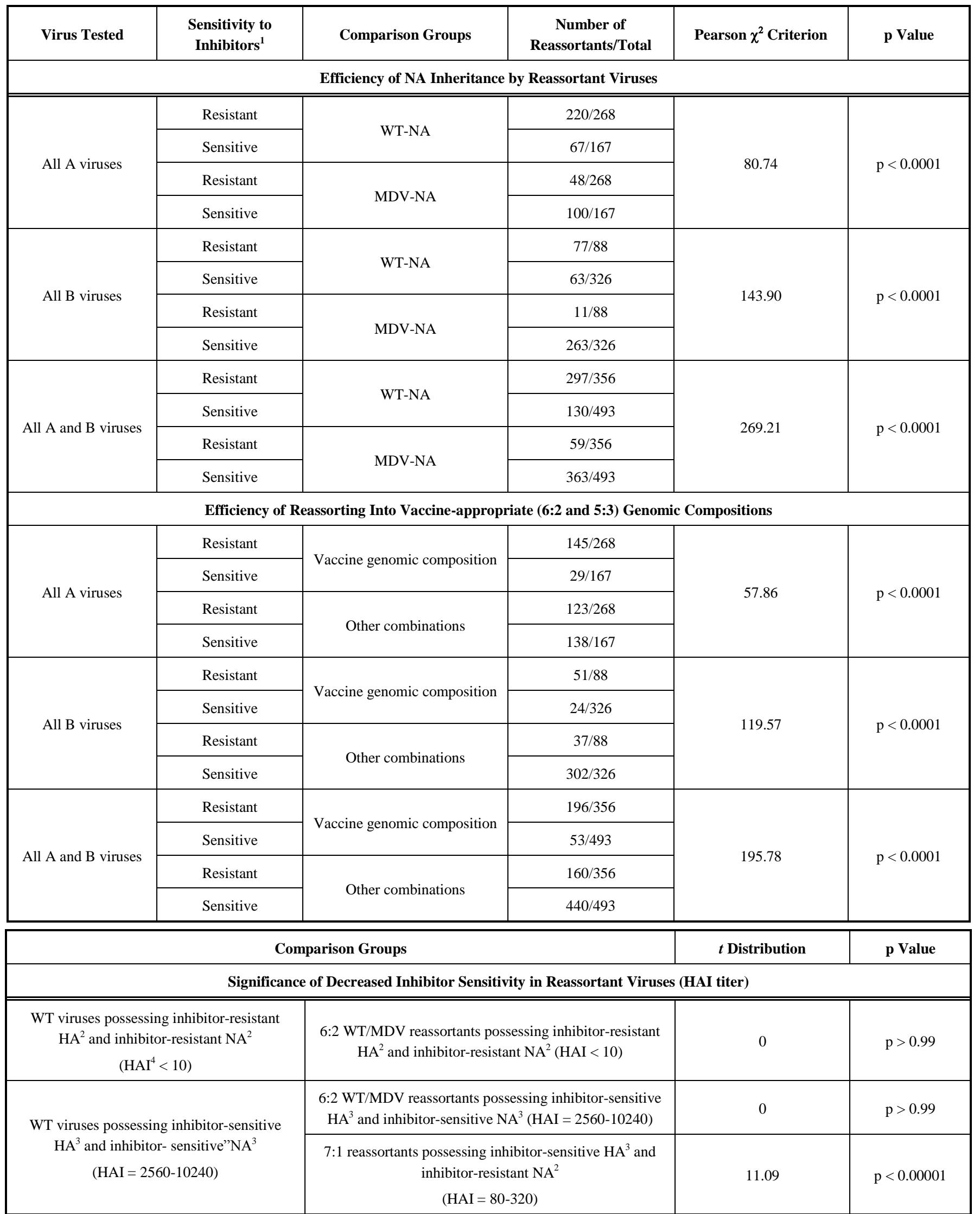

${ }^{1}$ Sensitivity to non-specific, heat-stable inhibitors in guinea pig serum.

${ }^{2}$ Gene derived from a virus resistant to non-specific, heat-stable inhibitors in guinea pig serum.

${ }^{3}$ Gene derived from a virus sensitive to non-specific, heat-stable inhibitors in guinea pig serum.

${ }^{4} \mathrm{HAI}$ : hemagglutination inhibition. 
Table 5. Sensitivity of influenza viruses to HAI by normal guinea pig sera.

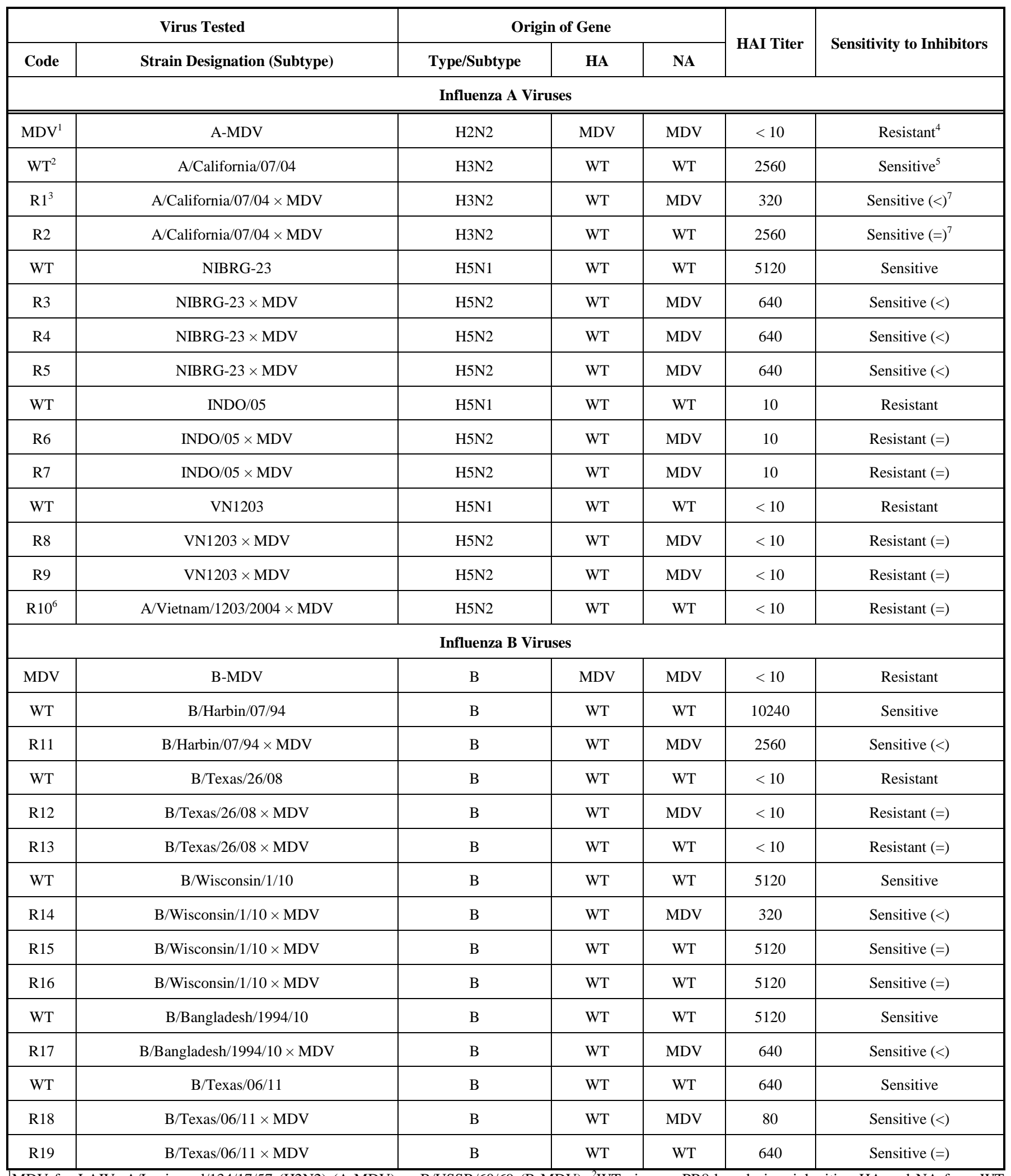

${ }^{1}$ MDV for LAIV: A/Leningrad/134/17/57 (H2N2) (A-MDV) or B/USSR/60/69 (B-MDV). ${ }^{2}$ WT virus or PR8-based virus inheriting HA and NA from WT virus. ${ }^{3}$ Reassortants of WT virus with MDV; the reassortants inherited HA from WT virus, and NA from WT virus (genomic composition 6:2) or MDV (genomic composition 7:1). ${ }^{4}$ Virus resistant to non-specific serum heat-stable inhibitors. ${ }^{5}$ Virus sensitive to non-specific serum heat-stable inhibitors. ${ }^{6} 6: 2$ R10 reassortant virus generated by plasmid-based reverse genetics as described in [14]. ${ }^{7}$ Sensitivity of reassortant virus to non-specific serum heat-stable inhibitors compared to WT parental virus; $\mathrm{p}<0.0001)$. 


\section{Reassortment of H5N1 Influenza Viruses with A-MDV for LAIV}

Highly pathogenic avian influenza viruses, having already overcome the species barrier in a number of cases, pose a potential pandemic threat. H5N1/PR8 candidates for inactivated influenza vaccines based upon viruses with pandemic potential are generated by reverse genetics methods by removing the multibasic amino acid motif associated with high pathogenicity in the HA gene and combining surface glycoprotein genes from avian virus with genes for internal proteins from A/Puerto Rico/8/34 (PR8) backbone. Recently, attempts have been made to use these attenuated H5N1/PR8 viruses as the source of HA and NA for classical reassortment with MDV to generate LAIV. However, mating A-MDV with either VN1203 or INDO/05 H5N1/PR8-based viruses has resulted in reassortants containing a single HA gene segment of the H5N1 influenza virus, and 7 RNA segments, including NA, from the AMDV. Another H5N1/PR8 virus, NIBRG-23, did not reassort with A-MDV at all.

In an attempt to disrupt the incredibly strong gene constellation of NIBRG-23, this virus was inactivated with ultraviolet light to lower the infectious titer by $6 \log _{10}$ effective infection dose $50\left(\mathrm{EID}_{50}\right) / \mathrm{mL}$ as previously described [15]. The inactivated virus was then used for coinfection with A-MDV in attempt of classical reassortment. One triple reassortant virus was generated in the initial coinfection, containing PB2, NP, and NS genes from PR8 virus, $\mathrm{PB} 1, \mathrm{PA}$, and $\mathrm{M}$ genes from $\mathrm{A}-\mathrm{MDV}$, and $\mathrm{HA}$ and NA genes from $\mathrm{H} 5 \mathrm{~N} 1$. We then back-crossed this progeny virus with A-MDV in order to obtain the desired 6:2 reassortant virus. However, we were unable to generate 6:2 H5N1 LAIV candidates: we isolated 3 cold-adapted and temperature sensitive reassortants from the back-cross, but they were of the 7:1 genotype. Thus, we did not detect any 6:2 reassortants with Leningrad backbone when using H5N1/PR8 viruses as the source of HA and NA.

Interestingly, we found a strong interaction between genes encoding avian NA and PB2 gene of PR8 virus in the genomes of H5N1/PR8 viruses. On the contrary, we also saw a balance between PB2/MDV and NA/MDV. Reassortants co-inherited the PB2/PR8 gene with NA/H5N1 gene, and PB2/MDV gene with NA/MDV gene (Table 7). We thus began a new series of experiments to determine the factors influencing co-inheritance of $\mathrm{PB} 2$ and NA genes of H5N1/PR8 reassortants. Table 6 shows the results of coinfecting A-MDV with PR8-based reassortant vaccine candidates for inactivated seasonal influenza vaccine of H3N2 (NIB-64) or H1N1 (IRV-148) subtypes. We isolated 28 reassortants based on IRV-148 (H1N1), and 9 reassortants based on NIB-64 (H3N2). This experiment indicated that all reassortants received WT NA. Additionally, 5/9 (55\%) NIB64-based reassortants and all 28 IRV-148-based reassortants inherited A-MDV PB2 gene. Moreover, 6 IRV-148-based reassortants and 2 NIB-64-based reassortants were 6:2.

Table 6. Sequence differences in HA and NA of single gene influenza virus reassortants.

\begin{tabular}{|c|c|c|c|c|c|c|c|c|}
\hline \multirow{2}{*}{\multicolumn{2}{|c|}{ Parental Viruses }} & \multicolumn{3}{|c|}{ Reassortants } & \multicolumn{4}{|c|}{ Substitutions in HA and NA Compared to Parental Virus } \\
\hline & & \multirow{2}{*}{ Code } & \multicolumn{2}{|c|}{$\begin{array}{l}\text { Origin of Gene } \\
\text { Encoding }\end{array}$} & \multicolumn{2}{|c|}{ Nt Change } & \multicolumn{2}{|c|}{ Amino Acid Change } \\
\hline WT or PR8-based virus & MDV & & HA & NA & HA & NA & HA1 & NA \\
\hline A/California/07/04 (H3N2) & A-MDV ${ }^{1}$ & $\mathrm{R} 1$ & WT & MDV & no change & no change & no change & no change \\
\hline NIBRG-23 (H5N1) & A-MDV & $\mathrm{R} 3$ & WT & MDV & no change & G-765-T & no change & Arg-249-Ile \\
\hline NIBRG-23 (H5N1) & A-MDV & $\mathrm{R} 4$ & WT & MDV & no change & no change & no change & no change \\
\hline NIBRG-23 (H5N1) & A-MDV & R5 & WT & MDV & no change & no change & no change & no change \\
\hline INDO/05 (H5N1) & A-MDV & R6 & WT & MDV & no change & no change & no change & no change \\
\hline INDO/05 (H5N1) & A-MDV & R7 & WT & MDV & no change & no change & no change & no change \\
\hline VN1203 (H5N1) & A-MDV & $\mathrm{R} 8$ & WT & MDV & no change & no change & no change & no change \\
\hline VN1203 (H5N1) & A-MDV & R9 & WT & MDV & $\begin{array}{c}\mathrm{T}-50-\mathrm{C} \\
\mathrm{T}-417-\mathrm{C}\end{array}$ & $\begin{array}{c}\text { G-765-T } \\
\text { no change }\end{array}$ & $\begin{array}{l}\text { no change } \\
\text { Ile-114-Thr }\end{array}$ & $\begin{array}{l}\text { Arg-249-Ile } \\
\text { no change }\end{array}$ \\
\hline B/Harbin/07/94 & B-MDV ${ }^{2}$ & R11 & WT & MDV & no change & G-1085-A & no change & no change \\
\hline B/Texas/26/08 & B-MDV & $\mathrm{R} 12$ & WT & MDV & no change & no change & no change & no change \\
\hline $\mathrm{B} /$ Wisconsin/1/10 & B-MDV & R14 & WT & MDV & no change & G-1359-A & no change & Glu-436-Lys \\
\hline B/Bangladesh/1994/10 & B-MDV & R17 & WT & MDV & no change & G-1085-A & no change & no change \\
\hline B/Texas/06/11 & B-MDV & $\mathrm{R} 18$ & WT & MDV & no change & G-1349-A & no change & Glu-436-Lys \\
\hline
\end{tabular}


Table 7. Origin of NA and PB2 genes of A/Leningrad/134/17/57 MDV reassortants with PR8-based strains for inactivated influenza vaccine.

\begin{tabular}{|c|c|c|c|c|c|}
\hline \multirow{3}{*}{$\begin{array}{c}\text { PR8-based Strain for } \\
\text { Inactivated Influenza } \\
\text { Vaccine }^{2}\end{array}$} & \multicolumn{5}{|c|}{ Reassortants ${ }^{1}$ of A/Leningrad/134/17/57 (H2N2) MDV with PR8-based Strains } \\
\hline & \multirow[t]{2}{*}{ Total } & \multicolumn{2}{|c|}{ PB2 Gene From } & \multicolumn{2}{|c|}{ NA Gene From } \\
\hline & & PR8 & $\mathbf{M D V}^{3}$ & WT virus & MDV \\
\hline NIBRG-23 (H5N1) ${ }^{4}$ & 17 & 0 & $17(100 \%)$ & 0 & $17(100 \%)$ \\
\hline INDO/05 (H5N1) & 11 & 0 & $11(100 \%)$ & 0 & $11(100 \%)$ \\
\hline \multirow{2}{*}{ VN1203 (H5N1) } & 28 & 0 & $28(100 \%)$ & 0 & $28(100 \%)$ \\
\hline & 14 & $14(100 \%)$ & 0 & $14(100 \%)$ & 0 \\
\hline IRV-148 (H1N1) & $28^{5}$ & 0 & $28(100 \%)$ & $28(100 \%)$ & 0 \\
\hline
\end{tabular}

${ }^{1}$ All reassortants contained HA from WT virus. The origin of PB1, PA, NP, M, and NS genes varied. ${ }^{2}$ PR8-based strains for inactivated influenza vaccine contain HA and NA from the appropriate WT virus, and the 6 internal genes from PR8 virus. ${ }^{3} \mathrm{MDV}$ : A/Leningrad/134/17/57 (H2N2). ${ }^{4}$ Reassortants were generated after co-infecting MDV and NIBRG-23 (H5N1) virus inactivated by ultraviolet light. ${ }^{5} 6 / 28$ IRV-148-based reassortants and ${ }^{6} 2 / 9$ NIB-64-based reassortants were 6:2.

\section{DISCUSSION}

Vaccines are considered the best available protection against influenza. Live attenuated influenza vaccines contain reassortant viruses expressing HA and NA gene segments from circulating WT viruses, and the internal genes from cold-adapted attenuated MDVs. A widely used classical method of making reassortant vaccine virus is based on coinfecting a WT strain and a cold-adapted MDV into embryonated chicken eggs. Theoretically, 256 potential genotypes could arise from the reassortment between 2 influenza viruses. To increase the number of reassortants possessing a vaccine-appropriate genomic composition, 2 selecting factors, anti-MDV serum and low incubating temperatures $\left(25-26^{\circ} \mathrm{C}\right)$, are used $[3,4]$.

LAIV strain development and characterization takes at least 2.5-3 months, and is a reasonably complicated and difficult process. Successful and fast generation and characterization of vaccine reassortants depends on numerous factors. In this study we show that most influenza A and B viruses that circulated in the 1990s readily reassorted with MDVs, and reassortants with the desired vaccine genotype were easy to generate. The majority of the resulting reassortants inherited all internal genes from MDVs and HA and NA from WT parental viruses. In contrast, vaccine strains based on most recently circulating viruses were much more difficult to develop. Only $11-14 \%$ of reassortants possessed NA from the WT parental virus, and the percentage of internal genes derived from MDVs was relatively low.

Analysis of 849 reassortants based on influenza viruses circulating in different years revealed that clones with an expected 6:2 vaccine genotype were isolated much more frequently when using WT parental viruses resistant to nonspecific serum inhibitors. Thus, over $80 \%$ of the reassortants based on inhibitor-resistant WT viruses inherited WT NA. In contrast, only $26 \%$ of the reassortants based on inhibitorsensitive WT viruses inherited WT NA. Using inhibitorresistant variants obtained from inhibitor-sensitive WT viruses recommended by WHO for influenza vaccines may help to solve the problem of reliably obtaining $6: 2$ reassortants.

Based on these findings, we wanted to determine the role of NA in the efficiency of influenza virus reassortment, and why inhibitor-sensitive WT viruses so rarely donate NA to reassortants. The receptor-binding activity of the influenza virus can be non-specifically inhibited by inhibitors present in normal sera, though the molecular mechanisms of serum resistance in influenza viruses are not well known. Previous studies demonstrated that HA plays an important role in inhibitor sensitivity of influenza H3N2 virus [8]. However, these data rely on the assumption that reassortant viruses inherit an inhibitor-sensitive phenotype with the HA of the inhibitor-sensitive parental virus. Gimsa et al. [9] demonstrated that NA may also play a role in inhibitor sensitivity.

In the present study, we investigated the sensitivity of single-gene reassortants to non-specific inhibitors, and compared this sensitivity to that of their parental viruses. We introduced HA into MDV genomes following a reassortment event with inhibitor-sensitive or inhibitor-resistant WT influenza viruses of subtypes $\mathrm{H} 3 \mathrm{~N} 2$ and $\mathrm{H} 5 \mathrm{~N} 1$, and of type B. Replacing the NA of an inhibitor-sensitive WT virus with the NA of an inhibitor-resistant MDV (reassortants R1, R3R5, R11, R14, R17, R18) led to significantly reduced serum inhibitor sensitivity of the resulting reassortant virus. These data indicate that NA plays a role in the sensitivity of influenza viruses to non-specific serum inhibitors. Thus, viral sensitivity to inhibitors seems to depend on a combination of unique properties of HA and NA. 
Many strains recently identified by the WHO for inclusion into seasonal influenza vaccines, including most influenza B viruses of the Yamagata lineage, are highly sensitive to non-specific serum inhibitors $[5,6]$. The standard protocol of developing reassortants LAIVs contains a step of treating anti-MDV serum with RDE to remove nonspecific inhibitors. However, even when anti-MDV serum was appropriately treated with RDE, it may still contain a residual amount of NA inhibitors [7, 16, 17], which could influence reassortment if the WT parental virus is inhibitorsensitive. Passaging reassortant progeny in the presence of anti-MDV serum is another step in the LAIV preparation protocol [3, 4]; thus, reassortants possessing the desired HA and NA of the inhibitor-sensitive WT parent might be nonspecifically suppressed because of residual anti-NA activity in the serum.

Earlier publications discussed whether LAIV reassortant candidates should be inhibitor-sensitive or resistant. Serum inhibitor-resistant LAIV candidates were thought to be more suitable for human use [10]. However, naturally occurring inhibitors of influenza virus HA activity have been identified both in various sera (including human sera) and in fluid secretions [7, 18-20]. Respiratory tract mucosa is not only the site of viral infection, but also the site of defense against infection. From this point of view, inhibitor-resistant vaccine viruses are more likely to bind to and enter the sensitive host cells than those derived from inhibitor-sensitive WT viruses, and may be better suited for use in humans.

The H5N1 avian influenza viruses are highly pathogenic and have already overcome the species barrier, causing justifiably serious concern of a new pandemic. A novel approach to developing potential pandemic LAIV has been described recently. The central idea was to develop LAIV candidates by using H5N1/PR8 viruses attenuated by reverse genetics as the source of HA and NA. However, the resulting reassortants possessed $\mathrm{H} 5 \mathrm{HA}$, while the 7 other genes came from A-MDV [21]. Neither were we successful in generating $6: 2$ or 5:3 H5N1 LAIV candidates by classical reassortment with the A-MDV in this study. Our study identifies influenza virus gene segments that appear to be incompatible for generating H5N1/PR8-MDV reassortants. For instance, we found a strong link between genomic segments encoding avian origin NA and PR8 virus PB2 gene. We also observed a functional balance between H5N1 HA, MDV NA, and MDV PB2. This strong constellation effect between gene segments of parental viruses may affect reassortment.

A little is known about an efficacy of reassortment in vitro or in vivo. Some pairs of viruses are difficult to reassort, as seen in attempts to generate avian-human reassortant viruses with the desired genomic composition. Subarrao et al. [22] obtained 6:2 reassortants of H3N2 human virus with gull influenza $\mathrm{A}$ virus, but the attempt to generate $6: 2$ reassortants of $\mathrm{H} 1 \mathrm{~N} 1$ human viruses with the same gull influenza A virus was not successful. The authors suggested that some influenza virus gene segments appear to be incompatible for generation of reassortants.

Indeed, reassortment, especially obtaining reassortants with desired gene combinations (6:2, for instance) is really rare event. In recent publication [23] interesting observation was made that pandemic "H1N1 virus could not be reproduced either in cell cultures or in pigs coinfected with North American triple reassortant H1N1 and Eurasian H1N1 swine influenza viruses."

Molecular basis of such restriction is still unknown. We can only speculate that the nature of this process depends on combination of as yet unknown factors.

In our experiments, NIBRG-23 (H5N1) virus did not reassort with A-MDV at all. Only inactivating NIBRG-23 by ultraviolet light prior to reassortment allowed generation of $7: 1 \mathrm{H} 5 \mathrm{~N} 2$ reassortants. INDO/05 and VN1203 did reassort with the resulting 7:1 reassortant progeny without inactivating the WT parental virus. It is difficult to fully understand the reasons for the difficulties in reassorting some H5N1 avian viruses with the A-MDV (H2N2) human strain.

The experimental inability to generate $6: 2 \mathrm{H} 5 \mathrm{~N} 1 \mathrm{LAIV}$ candidates by classical reassortment should not discourage the use of a 7:1 H5N2 potential pandemic vaccine in case of need. The presence of NA from cold-adapted MDV, together with genetically modified HA/H5N1, should provide additional attenuation of H5N2 LAIV candidate against the highly pathogenic H5N1 avian influenza virus. Our previous study of MDV-specific mutations revealed that reassortant viruses containing HA and NA genes from A-MDV, and the 6 other genes from its WT progenitor, were significantly more temperature-sensitive than WT virus itself (I. IsakovaSivak, personal communication). This finding indicates that the A-MDV NA gene alone or together with the HA gene can potentially contribute to the attenuated phenotype of the MDV. Therefore, including the MDV NA gene into a H5 vaccine strain may improve vaccine safety. Furthermore, according to the generally accepted concept, the anti-HA antibody response is the main contributor in protection of humans against influenza. The anti-NA antibody can modify the severity of the illness, but is not as crucial as anti-HA antibodies. Avian influenza viruses are not an exception. Immunizing chickens with HA alone or in combination with NA induced complete protection against challenge with the H5N1 avian influenza virus [24]. Thus, 7:1 H5N2 reassortants are promising candidates for further evaluation in humans.

\section{CONCLUSION}

In conclusion, our data suggest that NA plays an important role in the efficiency of reassortment of WT parental viruses with CA MDVs. We also demonstrated that NA contributes to the inhibitor-sensitive phenotype of influenza reassortant viruses and affects reassortment efficiency. Clearly, LAIV development would benefit from a better understanding of the genetic features and mechanisms of influenza virus serum resistance. Such studies must continue and expand to identify the mechanisms of serum sensitivity and gene constellations in the genome of H5N1/PR8 reassortants.

\section{CONFLICT OF INTEREST}

The authors confirm that this article content has no conflict of interest. 


\section{ACKNOWLEDGMENTS}

This work was partially supported by the Program for Appropriate Technologies in Health (PATH).

\section{REFERENCES}

[1] Development of new influenza vaccine strains and diagnostics. Introduced by Order of the Ministry of Health of Russian Federation \#156/29. May 7, 1998. [in Russian]. http://www.recipe.ru/docs/nd/print.php?id=1745 (accessed April 29, 2014).

[2] WHO Recommendations on the composition of influenza virus vaccines.

http://www.who.int/influenza/vaccines/virus/recommendations/en/i ndex.html (accessed April 29, 2014).

[3] Wareing MD, Marsh GA, Tannock GA. Preparation and characterization of attenuated cold-adapted influenza A reassortants derived from the $\mathrm{A} /$ Leningrad/134/17/57 donor strain. Vaccine 2002; 20: 2082-90.

[4] Cox NJ, Maassab HF, Kendal AP. Comparative studies of wildtype and cold-mutant (temperature-sensitive) influenza viruses: Nonrandom reassortment of genes during preparation of live virus vaccine candidates by recombination at $25{ }^{\circ} \mathrm{C}$ between recent $\mathrm{H} 3 \mathrm{~N} 2$ and H1N1 epidemic strains and cold-adapted A/Ann Arbor/6/60. Virology 1959; 97: 190-4.

[5] Larionova N, Kiseleva I, Isakova I, Litvinova O, Klimov A, Rudenko L. Naturally-occurring temperature-sensitive strains of influenza B virus. Proceedings of IVW-2004 Conference; 2004 9297; Lisbon, Portugal.

[6] Larionova NV, Kiseleva IV, Isakova IN, Litvinova OM, Rudenko LG. Phenotype of epidemic influenza B virus strains isolated in different years. [Article in Russian]. Vopr Virusol 2006; 5: 38-41.

[7] Križanová O, Rathová V. Serum inhibitors of mixoviruses. Curr Top Microbiol Immunol 1969; 47: 125-51.

[8] Matrosovich M, Gao P, Kawaoka Y. Molecular mechanisms of serum resistance of human influenza $\mathrm{H} 3 \mathrm{~N} 2$ virus and their involvement in virus adaptation in a new host. J Virol 1998; 72(8): 6373-80.

[9] Gimsa U, Grötzinger I, Gimsa J. Two evolutionary strategies of influenza viruses to escape host non-specific inhibitors: alteration of hemagglutinin or neuraminidase specificity. Virus Res 1996; 42(1-2): 127-35.

[10] Peetermans J. Live influenza virus vaccines and preparation thereof. U.S. patent 1976 3953592. 1976 April.

[11] Klimov A, Cox NJ. PCR restriction analysis of genome composition and stability of cold-adapted reassortants live influenza vaccines. J Virol Methods 1995; 52: 41-9.
[12] Kiseleva IV, Voeten JTM, Teley LCP, et al. Genome composition analysis of reassortant influenza viruses used in seasonal and pandemic live attenuated influenza vaccine. Mol Gen Microbiol Virol 2011; 26(4): 174-85.

[13] WHO Manual on animal influenza diagnosis and surveillance. 2002 edition. http://www.who.int/csr/resources/publications/ influenza/whocdscsrncs20025rev.pdf (accessed April 29, 2014).

[14] Gustin KM, Maines TR, Belser JA, et al. Comparative immunogenicity and cross-clade protective efficacy of mammalian cell-grown inactivated and live-attenuated $\mathrm{H} 5 \mathrm{~N} 1$ reassortant vaccines in ferrets. J Infect Dis 2011; 204(10): 1491-9.

[15] Rudneva IA, Timofeeva TA, Shilov AA, et al. Effect of gene constellation and post-reassortment amino acid change on the phenotypic features of H5 influenza virus reassortants. Arch Virol 2007; 152(6): 1139-45.

[16] Choppin PW, Tamm I. Studies of two kinds of virus particles which comprise influenza A2 virus strains. II. Reactivity with virus inhibitors in normal sera. J Exp Med 1960; 112: 921-44.

[17] Cohen A, Belyavin G. Hemagglutination inhibition of Asian influenza viruses: a new pattern of response. Virology 1959; 7(1): 59-74.

[18] Husseini RH, Sweet C, Collie MH, Smith H. The relation of interferon and nonspecific inhibitors to virus levels in nasal washes of ferrets infected with influenza viruses of differing virulence. Br J Exp Pathol 1981; 62(1): 87-93.

[19] Ryan-Poirier KA, Kawaoka Y. Distinct glycoprotein inhibitors of influenza A virus in different animal sera. J Virology 1991; 65(1): 389-95.

[20] White MR, Helmerhorst EJ. Multiple components contribute to ability of saliva to inhibit influenza viruses. Oral Microbiol Immunol 2009; 24: 18-24.

[21] Gambaryan AS, Lomakina NF, Boravleva EY, et al. Comparative safety, immunogenicity, and efficacy of several anti-H5N1 influenza experimental vaccines in a mouse and chicken models (Testing of killed and live H5 vaccine). Influenza Other Resp Viruses 2012; 6(3): 188-95.

[22] Subbarao K, Webster RG, Kawaoka Y, Murphy BR. Are there alternative avian influenza viruses for generation of stable attenuated avian-human influenza A reassortant viruses? Virus Res 1995; 39(2-3): 105-18.

[23] Ma W, Liu Q, Qiao C et al. North American triple reassortant and Eurasian H1N1 swine influenza viruses do not readily reassort to generate a 2009 pandemic H1N1-like virus. mBio 2014; 5(2): e00919-13.

[24] Nayak B, Kumar S, DiNapoli JM, et al. Contributions of the avian influenza virus HA, NA, and M2 surface proteins to the induction of neutralizing antibodies and protective immunity. J Virol 2010; 5: 2408-20.

\author{
Received: April 28, 2014 \\ Revised: May 19, 2014 \\ Accepted: May 19, 2014 \\ (C) Kiseleva et al.; Licensee Bentham Open.
}

This is an open access article licensed under the terms of the Creative Commons Attribution Non-Commercial License (http://creativecommons.org/licenses/ by-nc/3.0/) which permits unrestricted, non-commercial use, distribution and reproduction in any medium, provided the work is properly cited. 